\title{
INDEFINITE BOUNDARY VALUE PROBLEMS ON GRAPHS
}

\author{
SONJA CURRIE AND BRUCE A. WATSON
}

\begin{abstract}
We consider the spectral structure of indefinite second order boundary-value problems on graphs. A variational formulation for such boundary-value problems on graphs is given and we obtain both full and half-range completeness results. This leads to a max-min principle and as a consequence we can formulate an analogue of Dirichlet-Neumann bracketing and this in turn gives rise to asymptotic approximations for the eigenvalues.
\end{abstract}

Mathematics subject classification (2010): 34B09, 34B45, 34L10, 34L20.

Keywords and phrases: Differential Operators, Graphs, indefinite, half-range completeness, eigenvalue asymptotics.

\section{REFERENCES}

[1] S. Agmon, Lectures on elliptic boundary-value problems, Princeton, 1965.

[2] F. Ali Menmeti, A characterization of a generalized $C^{\infty}$-notion on nets, Integral Equations and Operator Theory 9 (1986), 753-766.

[3] J. Avron, Adiabatic quantum transport in multiply connected systems, Reviews of Modern Physics. 60 (1988), 873-915.

[4] R. BeAls, Indefinite Sturm-Liouville Problems and Half-Range Completeness, J. Differential Equations 56 (1985), 391-407.

[5] P. Binding, B. Najman, A variational principle in Krein space, Trans. Amer. Math. Soc. 342 (1994), 489-499.

[6] R. CARLSON, Adjoint and self-adjoint differential operators on graphs, Electronic J. Differential Equations 1998, 6 (1998), 1-10.

[7] E. A. Coddington, N. Levinson, Theory of ordinary differential equations, McGraw-Hill, New York, 1955.

[8] R. Courant, D. Hilbert, Methods of Mathematical Physics Volume I, Wiley-Interscience, New York, 1989.

[9] B. CURGUS, H. LANGER, A Krein space approach to symmetric ordinary differential operators with an indefinite weight function, J. Differential Equations 79 (1989), 31-61.

[10] S. CURRIE, Spectral theory of differential operators on graphs, PhD Thesis, University of the Witwatersrand, Johannesburg, 2006.

[11] S. CURRIE, B.A. WATSON, Dirichlet-Neumann bracketing for boundary-value problems on graphs, Elec. J. Diff. Eq 2005 (2005), 1-11.

[12] N. GerasimenKo, B. PAVlov, Scattering problems on non-compact graphs, Theoretical and Mathematical Physics 74 (1988), 230-240.

[13] V. Hutson, J.S. PYM, Applications of Functional Analysis and Operator Theory, Academic Press, 1980.

[14] I. Gohberg, P. LanCASter, L. Rodman, Indefinite Linear Algebra and Applications, Birkhäuser Basel-Boston-Berlin, 2005.

[15] P. Kuchment, Graph models for waves in thin structures, Waves Random Media 12 (2002), R1-R24.

[16] M. A. NAimark, Linear differential operators, part I, Frederick Ungar Publishing Co., New York, 1967.

[17] Yu. V. Pokornyi, V. L. Pryadiev, Some problems of the qualitative Sturm-Liouville theory on a spatial network, Russian Math. Surveys 59, 3 (2004), 515-552. 
[18] Yu. V. Pokornyi, V. L. Pryadiev, A. Al-Obeid, On the oscillation of the spectrum of a boundary-value problem on a graph, Math. Notes 60 (1997), 351-353.

[19] W. Rudin, Real and Complex analysis, New York: McGraw-Hill, 1965.

[20] R. E. Showalter, Hilbert Space Methods for Partial Differential Equations, Electron. J. Diff. Eqns., Monograph 01, 2001.

[21] M. SOLOMYAK, On the spectrum of the Laplacian on regular metric trees, Waves Random Media 14 (2004), S155-S171.

[22] J. VON BELOW, Sturm-Liouville eigenvalue problems on networks, Math. Methods Appl. Sci. 10 (1988), 383-395.

[23] J. Weidmann, Linear Operators in Hilbert Spaces, Springer-Verlag, 1980.

[24] H. F. Weinberger, Variational methods for eigenvalue approximation, Society for Industrial and Applied Mathematics, Philadelphia, 1974. 